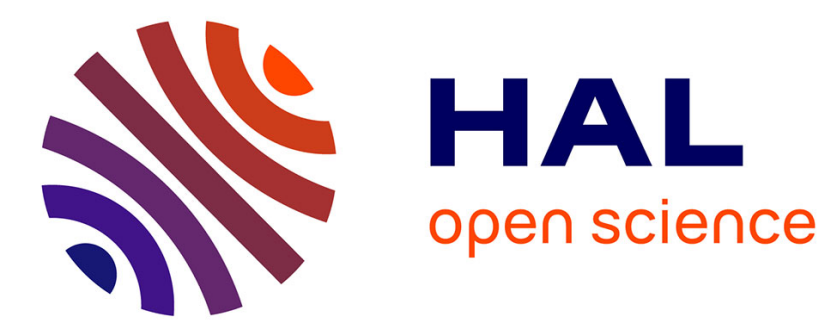

\title{
Enhanced Digital Signature using RNS Digit Exponent Representation
}

\author{
Thomas Plantard, Jean-Marc Robert
}

\section{To cite this version:}

Thomas Plantard, Jean-Marc Robert. Enhanced Digital Signature using RNS Digit Exponent Representation. WAIFI: Workshop on the Arithmetic of Finite Fields, Department of Mathematics of Ghent University, Jul 2016, Gand, Belgium. pp.177-192, 10.1007/978-3-319-55227-9_13 . hal-01337561

\section{HAL Id: hal-01337561 https://hal.science/hal-01337561}

Submitted on 27 Jun 2016

HAL is a multi-disciplinary open access archive for the deposit and dissemination of scientific research documents, whether they are published or not. The documents may come from teaching and research institutions in France or abroad, or from public or private research centers.
L'archive ouverte pluridisciplinaire $\mathbf{H A L}$, est destinée au dépôt et à la diffusion de documents scientifiques de niveau recherche, publiés ou non, émanant des établissements d'enseignement et de recherche français ou étrangers, des laboratoires publics ou privés. 


\title{
Enhanced Digital Signature using RNS Digit Exponent Representation
}

\author{
Thomas Plantard ${ }^{1}$, Jean-Marc Robert ${ }^{2,3}$ \\ 1 CCISR, SCIT, University of Wollongong, Australia \\ 2 Team DALI, Université de Perpignan Via Domitia, France \\ ${ }^{3}$ LIRMM, UMR 5506, Université Montpellier and CNRS, France
}

\begin{abstract}
Digital Signature Algorithm (DSA) involves modular exponentiation, of a public and known base by a random one-time exponent. In order to speed-up this operation, well-known methods take advantage of the memorization of base powers. However, due to the cost of the memory, to its small size and to the latency of access, previous research sought for minimization of the storage. In this paper, taking into account the modern processor features and the growing size of the cache memory, we improve the storage/efficiency trade-off, by using a RNS Digit exponent representation. We then propose algorithms for modular exponentiation. The storage is lower for equivalent complexities for modular exponentiation computation. The implementation performances show significant memory saving, up to 3 times for the largest NIST standardized key sizes compared to state of the art approaches.
\end{abstract}

Keywords: RNS, Digital Signature, Modular Exponentiation, Memory Storage, Efficient Software Implementation.

\section{Introduction}

In the DSS (Digital Signature Standard), DSA (Digital Signature Algorithm) is a popular authentication protocol. According to the NIST standard (see [3]), the public parameters are $p, q$ and $g$. The parameter $g$ is a generator of the multiplicative group $\mathbb{Z} / p \mathbb{Z}$ of order $q$, which is a prime of size corresponding to the required security level. Therefore, $p$ is a prime chosen such that $q$ divides $p-1$. The recommended security levels in the standard are 80-256 bits, corresponding to $160-512$ bit sizes for the prime $q$. When a server needs to sign a batch of documents or authentications, the main operations are modular exponentiations $g^{k} \bmod p$ (one per signature), where $k$ is a one time random parameter. Taking advantage of the fixed public parameter $g$ is a natural way to speed-up the signature protocol, by storing well chosen powers of $g$. The main known methods of the state of the art are the one presented by Gordon in [6], which stores the $g^{R^{i}} \bmod p$ values, and also the Fixed-base Comb, which is presented by Lim and Lee in [10]. While improving the complexity, and therefore, lowering the computation time, these methods require some storage. The trade-off between 
the efficiency and the storage amount is the comparison criteria between these different approaches.

All the protocols derived from the DSA can use these different approaches, since they all need an exponentiation of a known base by a random exponent. Blind signature and E-voting are examples of protocols using fixed-base modular exponentiation ([11]). Moreover, El-Gamal encryption and signature also use a public generator $g$ to the power of a randomly chosen exponent (see [4]). However, the decryption uses the result of this operation, and the idea does not apply in this case.

On the arithmetic side, the Residue Number System (RNS), based on the Chinese Remainder Theorem, is a classical way to speed-up and/or parallelize arithmetic computations and was first presented by Svoboda in [14] and by Garner in [5]. One can find a complete classical presentation of the RNS in Knuth [9].

Contributions: In this paper, we propose to use the memorization of base powers with numeration scales in radix $R=m_{0} \cdot m_{1}$ and the RNS representation of each digit using the base $\mathcal{B}=\left\{m_{0}, m_{1}\right\}$. We study the recoding algorithm and apply it to the exponent in modular exponentiation. We propose a modular exponentiation algorithm using this recoding of the exponent and memorization. We called this algorithm the $m_{0} m_{1}$ exponentiation method. We studied the corresponding complexities and storage amounts, and compared the results with the Fixed-base Comb and Radix- $R$ methods. We showed that our $m_{0} m_{1}$ exponentiation method has better storage/complexity trade-off that the aforementioned methods, for the NIST recommended field sizes and a large range of storage amount. We then made software implementations of our algorithms and performed tests in order to validate the storage/timing trade-off. The speed-up comparison shows the benefits. This approach provides also a fair flexibility in terms of required storage amount: one can choose the storage amount according to the device resources available and compatible to the global computation load of the system.

This paper is organized as follows: Section 2, we review the main classical fixed-base exponentiation algorithms, taking advantage of storage and give their complexities and storage requirements; Section 3, we present our approach for the $m_{0} m_{1}$ recoding; Section 4 , we then show the application on modular exponentiation; Section 5 shows the implementation strategies and results in terms of performances we got in this work; finally Section 6, we give some concluding remarks and perspectives.

\section{State of the Art Review}

In this Section, we review the state of the art classical approaches for fixedbase modular exponentiation.

When a server needs to sign a document or a message, the computation consists of several operations, the main one being a modular exponentiation 
$g^{k} \bmod p$, with $k$ being a one-time random exponent. This computation can use the classical Square-and-Multiply algorithm (see Algorithm 1). In terms of complexity, given the exponent length $t$ (that is, the size of the prime $q$ ), the number of modular squaring is $t-1$ and the number of modular multiplications to be computed is $t / 2$ on average, half of this length, for a randomly chosen exponent. There is no storage in this case.

The method presented by Gordon in [6] first suggests to store the $t$ successive squarings of $g$ (that is the sequence of $g^{2^{i}}$ ). In terms of complexity, given the exponent length $t$ (again, the size of the prime $q$ ), one has now no squarings and the number of modular multiplications to be computed is half of this length on average as in the previous case, for a randomly chosen exponent. The storage amount is $t$ values in $\mathbb{Z} / p \mathbb{Z}$ as mentioned above. Gordon in [6] mentions the generalization of this idea into a radix $R$ method, which consists of the memorization of the values $g^{i \cdot R^{j}}$, with $i \in[1, \ldots, R-1]$ and $0 \leq j<\ell$ where $\ell$ is the radix $R$ length of the exponent, which we denote by $w=\log _{2}(R)(\ell=\lceil t / w\rceil)$. In this case, the complexity is $\ell-1$ modular multiplications, for a storage amount of $\ell \cdot(R-1)$ values in $\mathbb{Z} / p \mathbb{Z}$. In the sequel, we will call this approach the Radix- $R$ Exponentiation Method (see Algorithm 2).
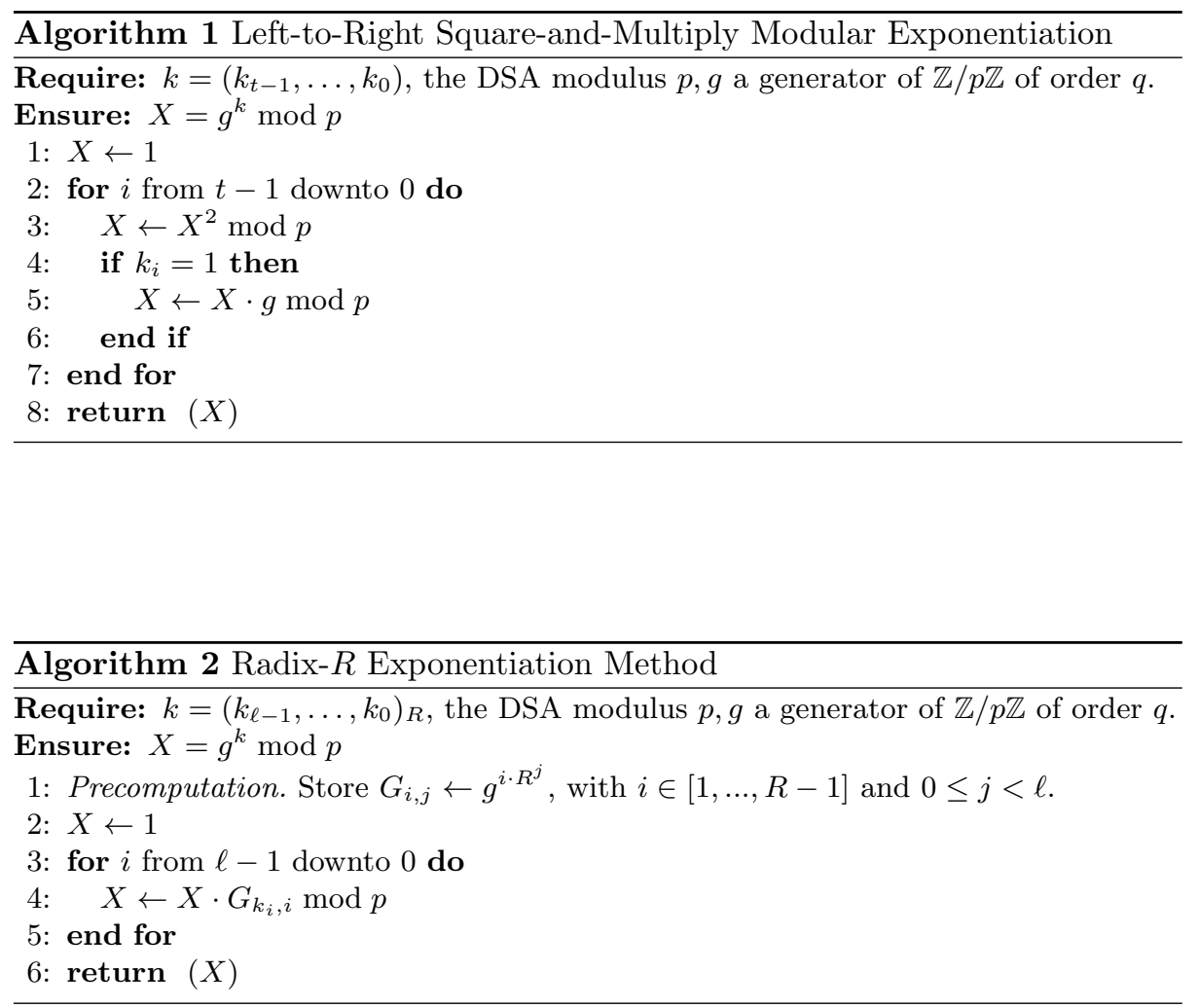


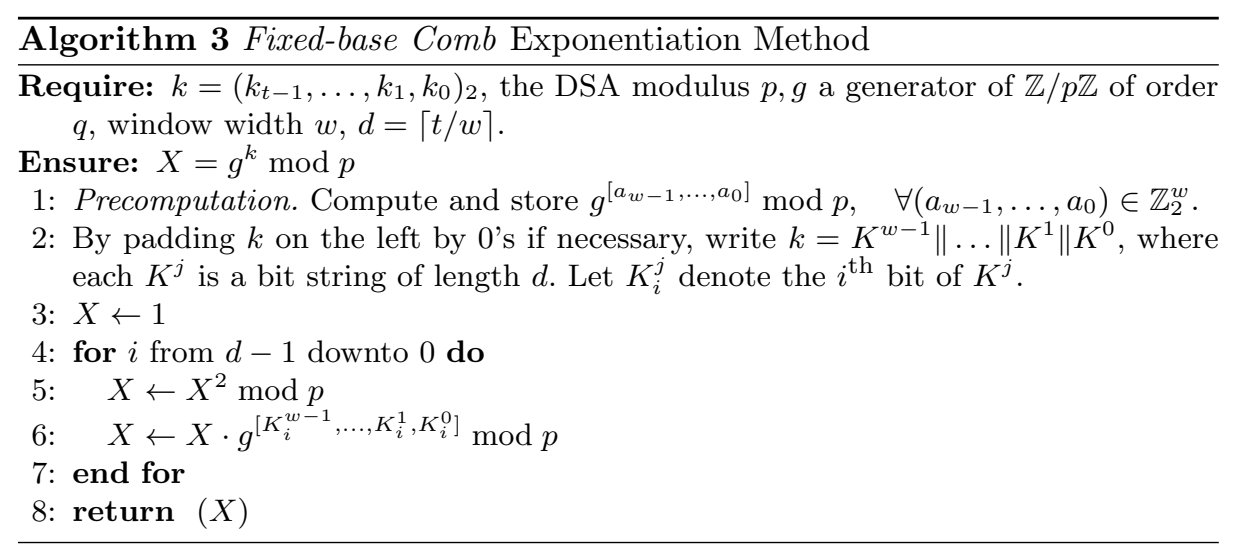

Another classical method is the so called Fixed-base Comb method. In [8], Hankerson et al. present this method proposed by Lim and Lee in [10]. The window width $w$ is the number of comb-teeth, and $d=\lceil t / w\rceil$ is the distance in bits between two teeth. This method is shown in Algorithm 3, in which we denote $\left[a_{w-1}, \ldots, a_{1}, a_{0}\right]=a_{w-1} 2^{(w-1) d}+\ldots+a_{2} 2^{2 d}+a_{1} 2^{d}+a_{0}$. The complexity of this approach is $d-1$ modular squarings and $d$ mutiplications, for a storage amount of $2^{w}-1$ values in $\mathbb{Z} / p \mathbb{Z}$. One drawback of this method is the lack of flexibility for the storage amount, which increases exponentially with respect to the window width $w$.

Table 1, we give the complexities and the storage amounts of all these approaches.

Table 1. Complexities and storage amounts of state of the art methods, average case, binary exponent length $t$. \#MM denotes the number of modular multiplications, \#MS the number of modular squarings.

\begin{tabular}{|c|c|c|c|}
\cline { 2 - 4 } \multicolumn{1}{c|}{} & $\#$ MM & $\#$ MS & storage $(\#$ values $\in \mathbb{Z} / p \mathbb{Z})$ \\
\hline Square-and-multiply, Algo. 1 & $t / 2$ & $t-1$ & - \\
\hline Radix- $R$ method, Algo. 2 & $\lceil t / w\rceil$ & - & $\lceil t / w\rceil \cdot(R-1)$ \\
\hline Fixed-base Comb, Algo 3 & $d=\lceil t / w\rceil$ & $d-1$ & $2^{w}-1$ \\
\hline
\end{tabular}

\section{$3 \quad m_{0} m_{1}$ Recoding}

In this section, we present our approach for the $m_{0} m_{1}$ recoding. Our goal is to use this representation in a modular exponentiation computation. The RNS digit representation with two moduli splits the digits in two parts. The first part will be used to select the precomputed values and the second part for final computation of the modular exponentiation, with the best possible trade-off. 


\subsection{Algorithm}

We first remind the RNS representation with RNS base $\mathcal{B}=\left\{m_{0}, m_{1}\right\}$ of two moduli. Let $R=m_{0} \cdot m_{1}$ and $x \in \mathbb{Z}$ such that $0 \leq x<R$. Let us also assume $m_{0}$ is prime, since this allows us to invert all integers $<m_{0}$ modulo $m_{0}$, and we choose $m_{1}<m_{0}$. In the sequel, we denote $|x|_{m}=x \bmod m$.

One represents $x$ with the residues

$$
\left\{\begin{array}{l}
x^{(0)}=|x|_{m_{0}} \\
x^{(1)}=|x|_{m_{1}}
\end{array}\right.
$$

and $x$ can be retrieved using the Chinese Remainder Theorem as follows:

$$
x=\left.\left|x^{(0)} \cdot m_{1} \cdot\right| m_{1}^{-1}\right|_{m_{0}}+\left.x^{(1)} \cdot m_{0} \cdot\left|m_{0}^{-1}\right|_{m_{1}}\right|_{R} .
$$

We now present our recoding approach. Our idea here is to use an exponent $k$ recoding in radix $R=m_{0} \cdot m_{1}$. We represent every radix- $R$ digits in RNS with RNS base $\mathcal{B}=\left\{m_{0}, m_{1}\right\}$. Let $k_{i}$ be the digits of $k$ in radix- $R$, and let us denote $\left(k_{i}^{(0)}, k_{i}^{(1)}\right)$ their RNS representations in base $\mathcal{B}$. Thus, one has:

$$
\begin{aligned}
& k=\sum_{i=0}^{\ell-1} k_{i} R^{i}, \text { with } \ell=\left\lceil t / \log _{2}(R)\right\rceil, \\
& \text { and }\left\{\begin{array}{l}
k_{i}^{(0)}=\left|k_{i}\right|_{m_{0}}, \\
k_{i}^{(1)}=\left|k_{i}\right|_{m_{1}} .
\end{array}\right.
\end{aligned}
$$

Let us denote (when $k_{i}^{(1)} \neq 0$ )

$$
\begin{aligned}
& m_{0}^{\prime}=m_{1} \cdot\left|m_{1}^{-1}\right|_{m_{0}}, \\
& m_{1}^{\prime}=m_{0} \cdot\left|m_{0}^{-1}\right|_{m_{1}}, \\
& k_{i}^{\prime}=\left|k_{i}^{(0)} \cdot\left(k_{i}^{(1)}\right)^{-1}\right|_{m_{0}} .
\end{aligned}
$$

One keeps $\kappa_{i} \leftarrow\left(k_{i}^{\prime}, k_{i}^{(1)}\right)$ as a representation of $k_{i}$ and this leads to $k_{i}=$ $\left|k_{i}^{(1)}\right| k_{i}^{\prime} \cdot m_{0}^{\prime}+\left.\left.m_{1}^{\prime}\right|_{R}\right|_{R}$. We handle the modular reduction $\bmod R$ as follows:

$$
k_{i}=k_{i}^{(1)}\left|k_{i}^{\prime} \cdot m_{0}^{\prime}+m_{1}^{\prime}\right|_{R}-\left\lfloor k_{i}^{(1)} \cdot\left|k_{i}^{\prime} \cdot m_{0}^{\prime}+m_{1}^{\prime}\right|_{R} / R\right\rfloor \cdot R .
$$

Let us denote $C=\left\lfloor k_{i}^{(1)} \cdot\left(k_{i}^{\prime} \cdot m_{0}^{\prime}+m_{1}^{\prime}\right) / R\right\rfloor$. By noticing that $0 \leq C<m_{1}$, we now consider $C$ as a carry that one can subtract to $k_{i+1}$. We then compute

if $k_{i+1} \geq C$ then $k_{i+1} \leftarrow k_{i+1}-C, C \leftarrow 0$, else $k_{i+1} \leftarrow k_{i+1}+R-C, C \leftarrow 1$, and one gets $k_{i+1} \geq 0$.

When $k_{i}^{(1)}=0$, we handle this by slightly rewriting $\kappa_{i}$ as follows: $\kappa_{i}=$ $\left(\left|k_{i}^{(0)}+1\right|_{m_{0}} \cdot m_{0}^{\prime}-m_{0}^{\prime}\right)$, thus keeping $\kappa_{i} \leftarrow\left(\left|k_{i}^{(0)}+1\right|_{m_{0}}, 0\right)$ as a representation of $k_{i}$ in this case. In addition, one notices that the carry $C$ is not modified here (it is either 0 or 1 and has been previously settled).

The sequence of the $\kappa_{i} \leftarrow\left(k_{i}^{\prime}, k_{i}^{(1)}\right)$ is the $m_{0} m_{1}$ recoding of $k$ we can use to compute a modular exponentiation.

One notices it might be necessary to process a last carry $C$, with a final correction. The recoding algorithm is shown in Algorithm 4. 


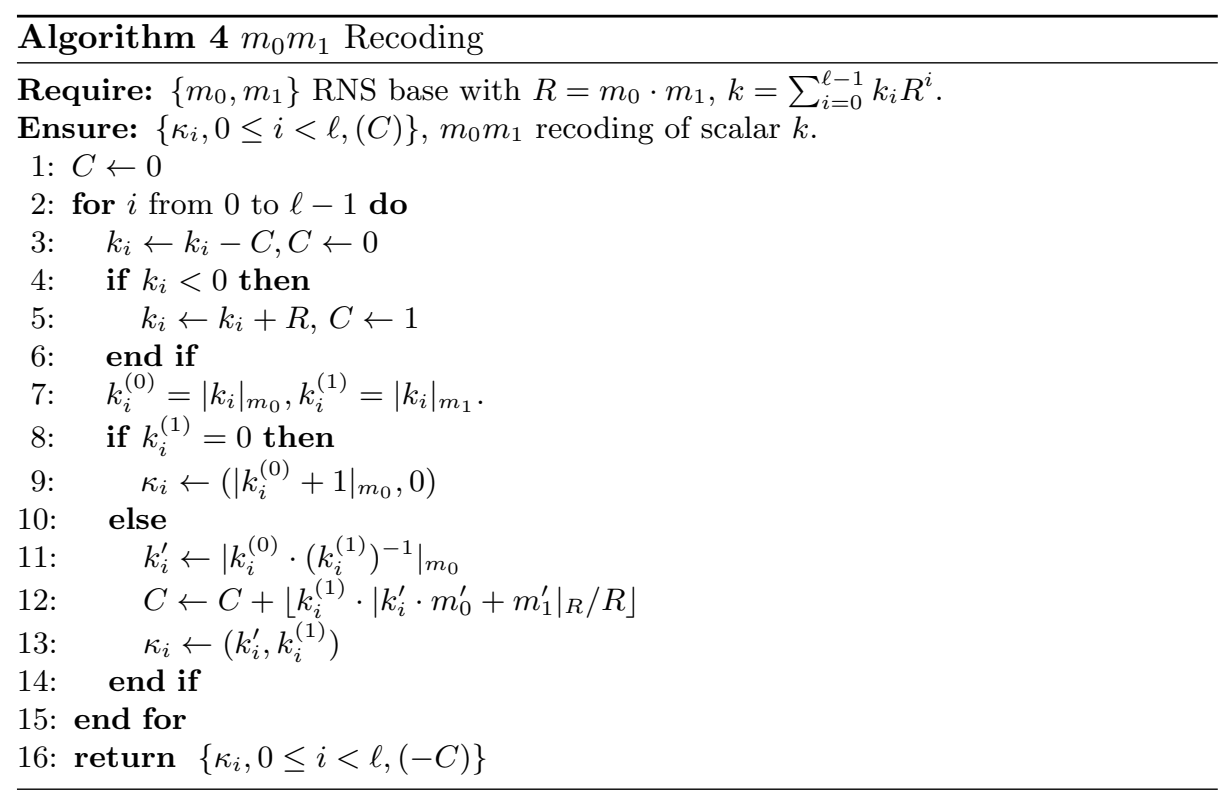

\section{$3.2 \quad$ Example}

We present here an example of $m_{0} m_{1}$ recoding with an exponent size $t$ of 20 bits $\left(0<k<2^{20}\right)$, and $\mathcal{B}=\{11,8\}$ (i.e. $\left.m_{0}=11, m_{1}=8\right)$. Thus, in this case, one has the radix $R=m_{0} \cdot m_{1}=88, \ell=\left\lceil 20 / \log _{2}(88)\right\rceil=4$, and therefore

$$
\begin{aligned}
& m_{0}^{\prime}=8 \cdot\left|8^{-1}\right|_{11}=56, \\
& m_{1}^{\prime}=11 \cdot\left|11^{-1}\right|_{8}=33 .
\end{aligned}
$$

Let us take $k=936192_{10}$, the random exponent. By rewriting $k$ in radix- $R$, one has

$$
k=48+78 \cdot 88+32 \cdot 88^{2}+1 \cdot 88^{3} .
$$

We now use Algorithm 4, which consists of a for loop, steps 2 to 15.

- In the first iteration $(i=0)$, one has $k_{0}=48$.

- One has $C \leftarrow 0$ and one skips the if-test steps 4 to 6 since $k_{0} \geq 0$.

- Step 7, one computes the RNS representation in base $\mathcal{B}$ of $k_{0}=48$ :

$$
k_{0}^{(0)}=\left|k_{0}\right|_{11}=4, k_{0}^{(1)}=\left|k_{0}\right|_{8}=0 .
$$

- Steps 6 and 7 , since $k_{0}^{(1)}=0$, one sets

$$
\kappa_{0} \leftarrow\left(\left|k_{0}^{(0)}+1\right|_{11}, 0\right)=(5,0)
$$

- In the second iteration $(i=1)$, one has $k_{1}=78$. 
- One has $C \leftarrow 0$ and one skips the if-test steps 4 to 6 since $k_{1} \geq 0$.

- Step 7, one computes the RNS representation in base $\mathcal{B}$ of $k_{1}=78$ :

$$
k_{1}^{(0)}=\left|k_{1}\right|_{11}=1, k_{1}^{(1)}=\left|k_{1}\right|_{8}=6 .
$$

- Steps 11 and 12 , since $k_{1}^{(1)} \neq 0$, one has

$$
\begin{array}{ll}
\left|\left(k_{1}^{(1)}\right)^{-1}\right|_{11} & \leftarrow 2 \\
k_{1}^{\prime}=\left|k_{1}^{(0)} \cdot\left(k_{1}^{(1)}\right)^{-1}\right|_{11} & \leftarrow 2 \\
C \leftarrow\left\lfloor\left(k_{1}^{(1)} \cdot\left|k_{1}^{\prime} \cdot 56+33\right|_{88}\right) / 88\right\rfloor & \leftarrow 3
\end{array}
$$

and finally

$$
\kappa_{1} \leftarrow(2,6)
$$

- In the third iteration $(i=2)$, one has now $k_{2} \leftarrow k_{2}-C=29$.

- The RNS representation in base $\mathcal{B}$ of $k_{2}$ is $k_{2}^{(0)}=7, k_{2}^{(1)}=5$.

- The computation steps 11-12 gives $C \leftarrow 2$, and

$$
\kappa_{2} \leftarrow(8,5)
$$

Without providing all the details, one finally gives the values returned by the algorithm:

$$
\kappa=((5,0),(2,6),(8,5),(3,7)), \text { and } C=-2 \text {. }
$$

\section{$4 m_{0} m_{1}$ Modular Exponentiation}

\subsection{Algorithm}

We now present the use of our recoding in the modular exponentiation. One wants to compute

$$
\begin{aligned}
g^{k} \bmod p & =g^{\sum_{i=0}^{\ell-1} k_{i} \cdot R^{i}} \bmod p \\
& =g^{\sum_{i=0}^{\ell-1} \kappa_{i} \cdot R^{i}} \cdot g^{C \cdot R^{\ell}} \bmod p \\
& =g^{C \cdot R^{\ell}} \cdot \prod_{i=0}^{\ell-1} g^{\kappa_{i} \cdot R^{i}} \bmod p
\end{aligned}
$$

with

$$
g^{\kappa_{i} \cdot R^{i}} \bmod p=g^{\kappa_{1}^{(1)} \cdot R^{i} \cdot\left|\kappa_{i}^{\prime} \cdot m_{0}^{\prime}+m_{1}^{\prime}\right|_{R}} \bmod p, \text { when } \kappa_{1}^{(1)} \neq 0
$$

and

$$
g^{\kappa_{i} \cdot R^{i}} \bmod p=g^{R^{i} \cdot\left|\kappa_{i}^{\prime} \cdot m_{0}^{\prime}+m_{1}^{\prime}\right|_{R}} \cdot g^{-R^{i} \cdot\left|m_{0}^{\prime}+m_{1}^{\prime}\right|_{R}} \bmod p, \text { when } \kappa_{1}^{(1)}=0 .
$$

In order to compute the fixed-base modular exponentiation $g^{k} \bmod p$, with $p$ prime, one stores the following values:

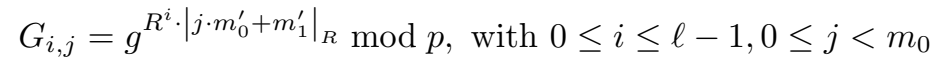




$$
\text { and } G_{\ell, 1}=g^{R^{\ell} \cdot\left|m_{0}^{\prime}+m_{1}^{\prime}\right|_{R} \bmod p .}
$$

The field inversion is very costly over $\mathbb{Z} / p \mathbb{Z}$, therefore, one also stores the following inverses:

$$
G_{i,-1}=g^{-\left|m_{0}^{\prime}+m_{1}^{\prime}\right|_{R} \cdot R^{i}} \bmod p \text { avec } 0 \leq i \leq \ell
$$

One uses one value $K_{j}$ per possible values of $1 \leq \kappa_{i}^{(1)}<m_{1}$, that is $m_{1}$ points. Thus, one now has

$$
K_{j}=\left(\prod_{\text {for all } \kappa_{i}^{(1)}=j} G_{i, \kappa_{i}^{(0)}}\right) \times\left(G_{\ell, \operatorname{sign}(C) 1}\right)_{|C|=j} \bmod p
$$

and

$$
K_{0}=\prod_{\text {for all } \kappa_{i}^{(1)}=0} G_{i, \kappa_{i}^{(0)}} \times G_{i,-1} \bmod p .
$$

This leads to

$$
g^{k} \bmod p=K_{0} \times \prod_{j=1}^{m_{1}} K_{j}^{j} .
$$

Every single individual modular exponentiation $K_{j}^{j}$ is performed with a square-and-multiply approach, which is more efficient than performing $j-1$ modular multiplications, even for small $m_{1}$.

One may notice that the amount of storage is now $\left(m_{0}+1\right) \times \ell+1$ points. This approach is depicted in Algorithm 5.

\subsection{Example}

We now go back to our previous example in Section 3.2 page 6 . One considers again the same values and parameters:

- an exponent size $t$ of 20 bits $\left(0<k<2^{20}\right)$, and $\mathcal{B}=\{11,8\}$

(i.e. $m_{0}=11, m_{1}=8$ );

- radix $R=m_{0} \cdot m_{1}=88(\ell=4)$;

- one has $k=936192_{10}$;

- and we use the $m_{0} m_{1}$ recoding previously computed:

$$
\kappa=((5,0),(2,6),(8,5),(3,7)) \text {, and } C=-2 .
$$

We present the computation of $g^{k} \bmod p$ using Algorithm 5. In terms of storage, one computes the values

$$
G_{i, j}=g^{R^{i} \cdot\left|j \cdot m_{0}^{\prime}+m_{1}^{\prime}\right|_{R}} \quad \bmod p \text { with } 0 \leq i \leq \ell-1 .
$$

One has the following values of $\left|j \cdot m_{0}^{\prime}+m_{1}^{\prime}\right|_{R}$ for $0 \leq j<11$ :

$$
\{33,1,57,25,81,49,17,73,41,9,65\}
$$




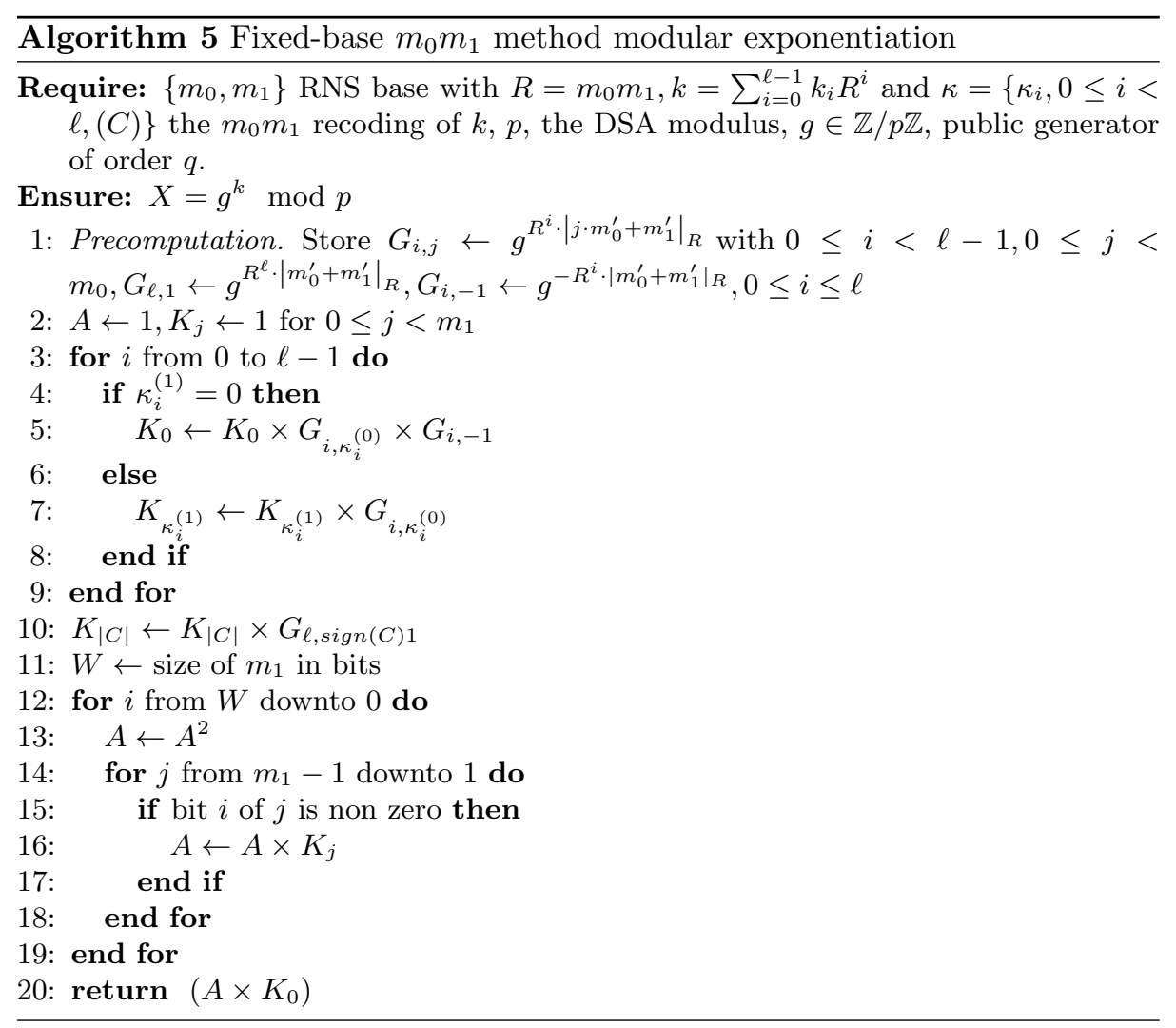

In our case, with the chosen parameters, this brings us to store the following values in $\mathbb{Z} / p \mathbb{Z}$ :

$G_{i}=\left\{g^{88^{i} \cdot 33}, g^{88^{i}}, g^{88^{i} \cdot 57}, g^{88^{i} \cdot 25}, g^{88^{i} \cdot 81}, g^{88^{i} \cdot 49}, g^{88^{i} \cdot 17}, g^{88^{i} \cdot 73}, g^{88^{i} \cdot 41}, g^{88^{i} \cdot 9}, g^{88^{i} \cdot 65}\right\}$.

We now use $\kappa$ in Algorithm 5.

- the first steps are a for loop (steps 3 to 9$)$ :

- in the first iteration, one has $\kappa_{0}^{(1)}=0$ (and $\kappa_{0}^{(0)}=5$ ), and this gives

$$
K_{0} \leftarrow G_{0, \kappa_{0}^{(0)}} \times G_{0,-1}=g^{49} \times g^{-1}=g^{48} .
$$

- in the second iteration, one has $\kappa_{1}^{(1)}=6\left(\right.$ and $\left.\kappa_{1}^{(0)}=2\right)$, and this gives

$$
K_{6} \leftarrow G_{1, \kappa_{1}^{(0)}}=g^{88 \cdot 57}=g^{5016} \text {. }
$$

- in the third iteration, one has $\kappa_{2}^{(1)}=5\left(\right.$ and $\left.\kappa_{2}^{(0)}=8\right)$, and this gives

$$
K_{5} \leftarrow G_{1, \kappa_{2}^{(0)}}=g^{88^{2} \cdot 41}=g^{317504} .
$$


- in the fourth and last iteration, one has $\kappa_{2}^{(1)}=7$ (and $\left.\kappa_{2}^{(0)}=3\right)$, and this gives

$$
K_{7} \leftarrow G_{1, \kappa_{2}^{(0)}}=g^{88^{3} \cdot 25}=g^{17036800} .
$$

- the last carry $C=-2$ is now processed (step 10):

$$
K_{2} \leftarrow G_{4,-1}=g^{88^{4} \cdot(-1)}=g^{-59969536} .
$$

- the reconstruction in the second for loop (steps 12 to 16) provides the final result by computing

$$
\begin{aligned}
g^{k} \bmod p & =K_{0} \times \prod_{j=1}^{m_{1}} K_{j}^{j} \bmod p \\
& =g^{48+2 \cdot(-59969536)+5 \cdot 317504+6 \cdot 5016+7 \cdot 17036800} \bmod p \\
& =g^{936192} \bmod p,
\end{aligned}
$$

which is the desired result.

\subsection{Complexity}

The complexity of Algorithm 5 is evaluated step by step in Table 2 for the average case. The number of field multiplications (MM) is evaluated as follows:

- the MMs in step 5 are performed only in case of $K_{0} \neq 1$, instead, it is only an instantiation of $K_{0}$;

- the MMs in step 7 are performed only in case of $K_{\kappa_{i}^{(1)}} \neq 1$, instead, it is only an instantiation of $K_{\kappa_{i}^{(1)}}$;

- the same applies for step 10 ;

This saves on average $m_{1}$ MMs, and this is taken into account in the Total line in Table 2 (it explains the $-m_{1}$ term). The number of operations in the final reconstruction is evaluated as follows:

- the modular squaring in step 13 is performed only in case of $A \neq 1$;

- the MMs in step 15 and 18 are performed only in case of $K_{j} \neq 1$;

For the sake of simplicity, we denote by $\mathcal{H}$ the sum of the $j$ Hamming weights for each $j$ from $m_{1}-1$ downto 1 (foreach loop step 14). The value of $\mathcal{H}$ is as follows for the different values of $m_{1}$ :

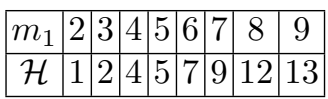

We now discuss the complexity comparison of the considered methods (Fixedbase Comb Algorithm 3, Radix-R Algorithm 2 and $m_{0} m_{1}$ Algorithm 3). Since the parameters are very different between these three methods, a formal comparison is difficult. Therefore, we present a comparison based on numerical application, for NIST recommended sizes. In the sequel of this section, we then provide complexity evaluations in terms of field multiplications MM, under the assumption 
Table 2. $m_{0} m_{1}$ modular exponentiation complexity and storage, average case.

\begin{tabular}{|c|c|c|}
\hline \multicolumn{3}{|c|}{ Complexity } \\
\hline Step & Operation & Complexity \\
\hline \hline$\ell / m_{1} \times$ step 5 & $K_{0} \leftarrow K_{0} \times G_{i,\left|\kappa_{i}^{(0)}+1\right|_{m_{0}}} \times G_{i,-1}$ & $2 \mathrm{MM}$ \\
\hline$\ell \frac{m_{1}-1}{m_{1}} \times$ step 7 & $K_{\kappa_{i}^{(1)}} \leftarrow K_{\kappa_{i}^{(1)}} \times G_{i, \kappa_{i}^{(0)}}$ & $1 \mathrm{MM}$ \\
\hline $1 \times$ step 10 & $K_{|C|} \leftarrow K_{|C|} \times G_{\ell, 1}^{s i n(C)}$ & $1 \mathrm{MM}$ \\
\hline$(W-1) \times$ step 13 & $A \leftarrow A^{2}$ & $1 \mathrm{MS}$ \\
\hline$(\mathcal{H}-1) \times$ steps 15 & $A \leftarrow A \times K_{j}$ & $1 \mathrm{MM}$ \\
\hline $1 \times$ step 18 & $\left(A \times K_{0}\right)$ & $1 \mathrm{MM}$ \\
\hline \hline TOTAL & $\left(\ell \frac{m_{1}+1}{m_{1}}-m_{1}+\mathcal{H}\right) \mathrm{MM}+(W-1) \mathrm{MS}$ \\
\hline TOTAL STORAGE & $\left(m_{0}+1\right) \times \ell+m_{1}+2$ elements of $\mathbb{Z} / p \mathbb{Z}$ \\
\hline
\end{tabular}

of squaring MS $=0.86 \mathrm{MM}$, which is the average value of our implementations for the NIST DSA recommended field sizes.

Figure 1 gives the general behavior of the three algorithms in terms of storage with respect to the complexity. One can see that the Fixed-base Comb method is the best for small storage amount. Our $m_{0} m_{1}$ approach is better for larger amount of storage, however, the Radix- $R$ method is the best when the storage is increasing. In the figure, the field size is the largest of the ones recommended in the NIST standards (see [13]). Thus, the storage amount for such size is very big. Nevertheless, the behavior is roughly the same for smaller sizes, although the benefit of our approach is lower. The NIST provides recommended key sizes and corresponding field size (respectively the size of the primes $q$ and $p$, see NIST SP800-57 [13]). This standardized sizes are as follows:

\begin{tabular}{|c|c|c|c|c|c|}
\hline key size (bits) & 160 & 224 & 256 & 384 & 512 \\
\hline field size (bits) & 1024 & 2048 & 3072 & 7680 & 15360 \\
\hline
\end{tabular}

For these sizes, Table 3 shows the complexity comparison between the Fixedbase Comb Algorithm 3, the Radix- $R$ Method Algorithm 2 and our $m_{0} m_{1}$ Recoding approach Algorithm 5. For an equivalent number of MMs, we provide the minimum amount of storage.

We now provide a few comments about this table.

- For all sizes, we do not provide the results for small amount of storage (values of $w<8$ ). For such storage, the Fixed-base Comb method is the best. One may notice that the Radix- $R$ approach needs the greatest storage at this complexity level.

- For intermediate values of complexity, our proposed $m_{0} m_{1}$ approach shows the best storage/complexity trade-off. However, the benefits are greater for the larger key sizes.

- $t=224$, the best gain of our $m_{0} m_{1}$ approach is for $\# M M \approx 24$, with a storage 5 to 8 times smaller than the storage required for the Fixed-base 
Fig. 1. Complexity comparison, Fixed base modular exponentiation NIST DSA, key size 512 bits (field size 15360 bits).

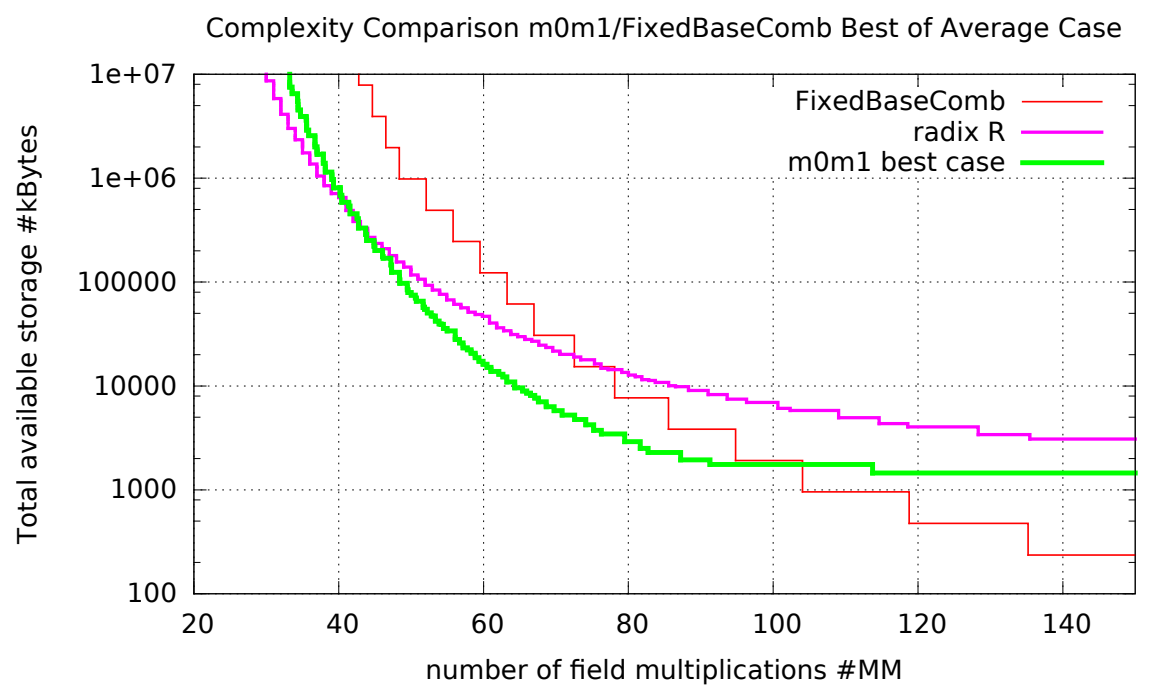

Comb method, respectively for $\# M M=30$ and $\# M M=24$, and $35 \%$ less than the one of the Radix- $R$ method. However, below $\# M M \approx 24$, the Radix- $R$ approach is better.

- $t=256$, the best gain of our $m_{0} m_{1}$ approach is for $\# M M \approx 32$, with a storage about 6 times smaller than the storage required for the Fixed-base $C o m b$ method, and $44 \%$ less than the one of the Radix- $R$ method. Again, with decreasing values of \#MM (below 26), the Radix- $R$ approach is better.

- $t=384$, the best gain of our $m_{0} m_{1}$ approach is for $\# M M \approx 35$, with a storage about 15 times smaller than the storage required for the Fixedbase Comb method, and $19 \%$ less than the one of the Radix- $R$ method. Again, with decreasing values of $\# M M$ (below 33), the $\operatorname{Radix}-R$ approach is better.

- $t=512$, the best gain of our $m_{0} m_{1}$ approach is for $\# M M \approx 41$, with a storage about 22 times smaller than the storage required for the Fixedbase Comb method, and $27 \%$ less than the one of the Radix- $R$ method. Again, with decreasing values of \#MM (below 38), the $\operatorname{Radix}-R$ approach is better.

However, one may notice that the bigger memory storage sizes exceed the common values of Random Access Memory, and also the maximum allowed for the malloc function of the standard C library for memory allocation. Neverthe- 
Table 3. Storage amount comparison, Fixed-base Comb method and $m_{0} m_{1}$ modular exponentiation fixed-base, average case, NIST recommended exponent sizes.

\begin{tabular}{|c|c|c|c|}
\hline \multicolumn{4}{|c|}{ Key size $t=224$ bits } \\
\hline$\# M M$ & \begin{tabular}{|l|l} 
& Fixed-base $C$ \\
\end{tabular} & \begin{tabular}{l|l} 
X. & Radix- $R$ \\
\end{tabular} & $m_{0} m_{1}$ \\
\hline \multirow{2}{*}{45} & $127.5 \mathrm{kB}$ & $345 \mathrm{kB}$ & $108 \mathrm{kB}$ \\
\hline & $w=9$ & $R=31$ & $m_{0}=11 ; m_{1}=9$ \\
\hline \multirow{2}{*}{37} & $511.5 \mathrm{kB}$ & $594 \mathrm{kB}$ & $242 \mathrm{kB}$ \\
\hline & $w=11$ & $R=61$ & $31 ; 7$ \\
\hline \multirow{2}{*}{30} & $4095.5 \mathrm{kB}$ & $1386 \mathrm{kB}$ & $770 \mathrm{kB}$ \\
\hline & $w=14$ & $R=179$ & $127 ; 7$ \\
\hline \multirow{2}{*}{24} & $32767.5 \mathrm{kB}$ & $4230 \mathrm{kB}$ & $4173 \mathrm{kB}$ \\
\hline & $w=17$ & $R=677$ & $877 ; 7$ \\
\hline \multirow{2}{*}{19} & $524287.5 \mathrm{kB}$ & $27084 \mathrm{kB}$ & $50409 \mathrm{kB}$ \\
\hline & $w=21$ & $R=5417$ & $13441 ; 5$ \\
\hline \multicolumn{4}{|c|}{ Key size $t=384$ bits } \\
\hline$\# M M$ & Fixed-base $C$. & Radix- $R$ & $m_{0} m_{1}$ \\
\hline \multirow{2}{*}{63} & $1918 \mathrm{kB}$ & $4081 \mathrm{kB}$ & $969 \mathrm{kB}$ \\
\hline & $w=11$ & $R=67$ & $m_{0}=19 ; m_{1}=1$ \\
\hline \multirow{2}{*}{50} & $15358 \mathrm{kB}$ & $10087 \mathrm{kB}$ & $3742 \mathrm{kB}$ \\
\hline & $w=14$ & $R=191$ & $101 ; 11$ \\
\hline \multirow[t]{2}{*}{41} & $122878 \mathrm{kB}$ & $26655 \mathrm{kB}$ & $17284 \mathrm{kB}$ \\
\hline & $w=17$ & $R=677$ & $541 ; 6$ \\
\hline \multirow{2}{*}{35} & $983038 \mathrm{kB}$ & $80357 \mathrm{kB}$ & $64768 \mathrm{kB}$ \\
\hline & $w=20$ & $R=2381$ & $2381 ; 6$ \\
\hline \multirow{2}{*}{30} & $7864318 \mathrm{kB}$ & $246070 \mathrm{kB}$ & $315053 \mathrm{kB}$ \\
\hline & $w=23$ & $R=8467$ & $13441 ; 5$ \\
\hline \multirow{2}{*}{26} & $62914558 \mathrm{kB}$ & $951217 \mathrm{kB}$ & $3256278 \mathrm{kB}$ \\
\hline & $w=26$ & $R=37579$ & $165397 ; 5$ \\
\hline \multirow{2}{*}{24} & $503316478 \mathrm{kB}$ & $1750756 \mathrm{kB}$ & $-\mathrm{kB}$ \\
\hline & $w=29$ & $R=74699$ & - \\
\hline
\end{tabular}

\begin{tabular}{|c|c|c|c|}
\hline \multicolumn{4}{|c|}{ Key size $t=256$ bits } \\
\hline$\# M M$ & Fixed-base $C$ & Radix- $R$ & $m_{0} m_{1}$ \\
\hline \multirow{2}{*}{46} & $383 \mathrm{kB}$ & $845 \mathrm{kB}$ & $241 \mathrm{kB}$ \\
\hline & $w=10$ & $R=47$ & $m_{0}=17 ; m_{1}=11$ \\
\hline \multirow{2}{*}{39} & $1535 \mathrm{kB}$ & $1454 \mathrm{kB}$ & $579 \mathrm{kB}$ \\
\hline & $w=12$ & $R=97$ & $47 ; 7$ \\
\hline \multirow{2}{*}{32} & $12287 \mathrm{kB}$ & $3179 \mathrm{kB}$ & $2070 \mathrm{kB}$ \\
\hline & $w=15$ & $R=257$ & $211 ; 6$ \\
\hline \multirow{2}{*}{26} & $98303 \mathrm{kB}$ & $9486 \mathrm{kB}$ & $9642 \mathrm{kB}$ \\
\hline & $w=18$ & $R=937$ & $1223 ; 6$ \\
\hline \multirow{2}{*}{20} & $1572863 \mathrm{kB}$ & $66676 \mathrm{kB}$ & $225482 \mathrm{kB}$ \\
\hline & $w=22$ & $R=8467$ & $37579 ; 5$ \\
\hline \multicolumn{4}{|c|}{ Key size $t=512$ bits } \\
\hline$\# M M$ & Fixed-base C. & Radix- $R$ & $m_{0} m_{1}$ \\
\hline \multirow{2}{*}{86} & $3836 \mathrm{kB}$ & $9841 \mathrm{kB}$ & $1940 \mathrm{kB}$ \\
\hline & $w=11$ & $R=59$ & $m_{0}=13 ; m_{1}=11$ \\
\hline \multirow{2}{*}{73} & $15356 \mathrm{kB}$ & $17855 \mathrm{kB}$ & $4747 \mathrm{kB}$ \\
\hline & $w=13$ & $R=127$ & $41 ; 10$ \\
\hline \multirow{2}{*}{60} & $122876 \mathrm{kB}$ & $46775 \mathrm{kB}$ & $16224 \mathrm{kB}$ \\
\hline & $w=16$ & $R=409$ & $179 ; 11$ \\
\hline \multirow{2}{*}{52} & $491516 \mathrm{kB}$ & $93110 \mathrm{kB}$ & $54680 \mathrm{kB}$ \\
\hline & $w=18$ & $R=937$ & $677 ; 7$ \\
\hline \multirow{2}{*}{48} & $983036 \mathrm{kB}$ & $156091 \mathrm{kB}$ & $106185 \mathrm{kB}$ \\
\hline & $w=19$ & $R=1699$ & $1489 ; 10$ \\
\hline \multirow{2}{*}{41} & $7864316 \mathrm{kB}$ & $489112 \mathrm{kB}$ & $355573 \mathrm{kB}$ \\
\hline & $w=22$ & $R=6211$ & $5417 ; 7$ \\
\hline \multirow[t]{2}{*}{35} & $62914556 \mathrm{kB}$ & $2048419 \mathrm{kB}$ & $2113890 \mathrm{kB}$ \\
\hline & $w=25$ & $R=30347$ & $37579 ; 7$ \\
\hline
\end{tabular}

less, the storage savings proposed by our method and the Radix- $R$ ones allow to keep the level under the limit for lower complexities.

As a conclusion, our $m_{0} m_{1}$ approach shows lower storage amount for intermediate values of storage, whatever the standardized key size.

\section{$5 \quad$ Implementation results}

\subsection{Implementation strategies}

We review hereafter the main implementation strategies and test process. This applies for the three considered exponentiation algorithms. The algorithms were coded in C, compiled with gcc 4.8.3 and run on the same platform.

Multiprecision Multiplication and Squaring: we used the low level functions performing multi-precision multiplication and squaring of the GMP library as building blocks of our codes (GMP 6.0.0, see GMP library [1]). According to the GMP documentation, the classical schoolbook algorithm is used for small sizes, and Karatsuba and Toom-Cook sub quadratic methods for size $\geq 2048$ bits.

Modular Reduction: this operation implements the Montgomery representation and modular reduction method, which avoid multi-precision division in the computation of the modular reduction. This approach has been presented by Mont- 
gomery in [12]. More specifically, we used the block Montgomery algorithm suggested by Bosselaers et al. in [2]. In this algorithm, the multi-precision operations combine full size operand with one word operand and are also available in the GMP library [1]. Although the complexity is the same, the implementation is more computer friendly.

$m_{0} m_{1}$ Recoding: the conversion in radix- $R$ needs multi-precision divisions. These operations are implemented using the GMP library [1]. The size of these operations is decreasing along the algorithm, and this is managed through GMP. The other operations are classical long integer operations. Steps 9 and 21 in Algorithm 4, an inversion modulo $m_{0}$ is required $\left(\left|\left(k_{i}^{(1)}\right)^{-1}\right|_{m_{0}}\right)$. This operation is performed using the Extended Euclidean Algorithm, over long integer data. For the considered exponent sizes, the cost of the recoding is negligible. This is explained by the small size of the exponent in comparison with the size of the data processed during the modular exponentiation (see the key sizes given page 11). The timings given in the next Section include this recoding.

Test processing : the tests involve a few hundred dataset, which consists of random exponent inputs and an exponentiation base with the precomputed values stored. We compute 2000 times the corresponding exponentiation for each dataset and keep the minimum number of clock cycles. This avoids the cold-cache effect and system issues. The timings are obtained by averaging the timings of all dataset.

\subsection{Tests results and comparison}

The three considered exponentiation algorithms were coded in C, compiled with gcc 4.8.3 and run on the following platform: the CPU is an Intel XEON ${ }^{\circledR}$ E5-2650 (Ivy bridge), and the operating system is CENTOS 7.0.1406. On this platform, the Random Access Memory is 12.6 GBytes. One notices that the performance results include the recoding in the radix- $R$ and $m_{0}, m_{1}$ cases. The implementation results confirm the complexity evaluation, for key sizes of 224 , 256, 384, and 512 bits. However, the better results are for 384 and 512 bits.

In Table 4, we provide the most significant results. The gains shown are roughly in the same order of magnitude as the one of the complexity evaluation. In particular, for the largest key size (512 bits), the storage of our $m_{0}, m_{1}$ approach is nearly ten times less than the one required with the Fixed-base Comb method, and nearly $14 \%$ less than the one required for the Radix- $R$ method, for the same computation timing, about $12.5 \times 10^{6} \# C C$.

\section{Conclusion and future work}

In this paper, we have presented a new method for fixed-base exponentiation using a radix- $R$ conversion with RNS representation of every radix- $R$ digits, using an RNS base with two moduli $\mathcal{B}=\left\{m_{0}, m_{1}\right\}$. We have designed a recoding algorithm, which computes our $m_{0} m_{1}$ representation of the exponent, and 
Table 4. Synthesis of implementation results, clock cycles and storage $(\mathrm{kB})$. Test performed on an Intel XEON E5-2650 (Ivy bridge), gcc 4.8.3, CENTOS 7.0.1406.

\begin{tabular}{|c|c|c|c|}
\hline \multicolumn{4}{|c|}{ Modular Exponentiation } \\
\hline $\begin{array}{r}\text { State of the } \\
\text { Fixed-base Comb }\end{array}$ & $\begin{array}{r}\text { Art methods } \\
\text { radix } R\end{array}$ & $m_{0}, m_{1}$ rec. & ratio \\
\hline $\begin{array}{c}\# C C \\
\text { Storage }\end{array}$ & $\begin{array}{c}\# C C \\
\text { Storage }\end{array}$ & $\begin{array}{c}\# C C \\
\text { Storage }\end{array}$ & $\begin{array}{c}m_{0}, m_{1} \\
\text { /Best S.o.A. }\end{array}$ \\
\hline \multicolumn{4}{|c|}{ key size 224 bits, field size 2048 bits (level of security: 112 bits) } \\
\hline $\begin{array}{c}221108 \\
1023.5 \mathrm{kB}(w=12)\end{array}$ & $\begin{array}{c}227838 \\
829 \mathrm{kB}(R=91)\end{array}$ & 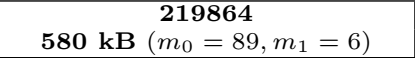 & $\begin{array}{l}\times 0.994 \\
\times 0.700\end{array}$ \\
\hline $\begin{array}{c}210074 \\
2047.5 \mathrm{kB}(w=13)\end{array}$ & $\begin{array}{c}206888 \\
1324 \mathrm{kB}(R=163)\end{array}$ & $\begin{array}{c}\text { 207072 } \\
766 \mathbf{~ k B}\left(m_{0}=127, m_{1}=7\right)\end{array}$ & $\begin{array}{l}\times 0.985 \\
\times 0.579\end{array}$ \\
\hline $\begin{array}{c}149690 \\
65535 \mathrm{kB}(w=18) \\
\end{array}$ & $\begin{array}{c}147877 \\
\mathbf{7 2 8 9} \mathbf{k B}(R=1223)\end{array}$ & $\begin{array}{c}146156 \\
21599 \mathrm{kB}\left(m_{0}=5417, m_{1}=6\right) \\
\end{array}$ & $\begin{array}{l}\times 0.988 \\
\times 2.96 \\
\end{array}$ \\
\hline \multicolumn{4}{|c|}{ key size 256 bits, field size 3072 bits (level of security: 128 bits) } \\
\hline $\begin{array}{c}524539 \\
1535 \mathrm{kB}(w=12)\end{array}$ & $\begin{array}{c}502981 \\
1411 \mathrm{kB}(R=91)\end{array}$ & $\begin{array}{c}\mathbf{5 0 1 4 6 6} \\
\mathbf{8 9 7} \mathbf{k B}\left(m_{0}=79, m_{1}=6\right)\end{array}$ & $\begin{array}{l}\times 0.997 \\
\times 0.636 \\
\end{array}$ \\
\hline $\begin{array}{c}449397 \\
6143 \mathrm{kB}(w=14)\end{array}$ & $\begin{array}{c}445871 \\
2251 \mathrm{kB}(R=163)\end{array}$ & $\begin{array}{c}446444 \\
2056 \mathrm{kB}\left(m_{0}=211, m_{1}=6\right)\end{array}$ & $\begin{array}{l}\times 1.001 \\
\times 0.913 \\
\end{array}$ \\
\hline $\begin{array}{c}356892 \\
98303 \mathrm{kB}(w=18) \\
\end{array}$ & $\begin{array}{c}354640 \\
\mathbf{6 4 1 4} \mathrm{kB}(R=571) \\
\end{array}$ & $\begin{array}{c}354071 \\
12843 \mathrm{kB}\left(m_{0}=1721, m_{1}=7\right) \\
\end{array}$ & $\begin{array}{l}\times 0.998 \\
\times 2.002 \\
\end{array}$ \\
\hline \multicolumn{4}{|c|}{ key size 384 bits, field size 7680 bits (level of security: 192 bits) } \\
\hline $\begin{array}{c}4442590 \\
1918 \mathrm{kB}(w=11)\end{array}$ & $\begin{array}{c}4492191 \\
3430 \mathrm{kB}(R=53)\end{array}$ & $\begin{array}{c}\mathbf{4 4 0 9 5 8 4} \\
1134 \mathrm{kB}\left(m_{0}=23, m_{1}=10\right)\end{array}$ & $\begin{array}{l}\times 0.993 \\
\times 0.467\end{array}$ \\
\hline $\begin{array}{c}3554339 \\
15358 \mathrm{kB}(w=14)\end{array}$ & $\begin{array}{c}\mathbf{3 5 2 4 8 9 6} \\
8290 \mathrm{kB}(R=163)\end{array}$ & $\begin{array}{c}3551437 \\
\mathbf{4 1 6 4} \mathbf{~ k B}\left(m_{0}=113, m_{1}=10\right)\end{array}$ & $\begin{array}{l}\times 1.008 \\
\times 0.502\end{array}$ \\
\hline $\begin{array}{c}2736341 \\
245758 \mathrm{kB}(w=18) \\
\end{array}$ & $\begin{array}{c}\mathbf{2 5 4 3 4 8 0} \\
45221 \mathrm{kB}(R=1223) \\
\end{array}$ & $\begin{array}{c}2743399 \\
\mathbf{2 9 9 6 1} \mathbf{k B}\left(m_{0}=1031, m_{1}=7\right) \\
\end{array}$ & $\begin{array}{l}\times 1.079 \\
\times 0.662 \\
\end{array}$ \\
\hline \multicolumn{4}{|c|}{ key size 512 bits, field size 15360 bits (level of security: 256 bits) } \\
\hline $\begin{array}{c}18632429 \\
15536 \mathrm{kB}(w=13)\end{array}$ & $\begin{array}{c}19260731 \\
13765 \mathrm{kB}(R=91)\end{array}$ & $\begin{array}{c}18550238 \\
4745 \mathrm{kB}\left(m_{0}=41, m_{1}=10\right)\end{array}$ & $\begin{array}{l}\times 0.996 \\
\times 0.345\end{array}$ \\
\hline $\begin{array}{c}14848261 \\
122876 \mathrm{kB}(w=16) \\
\end{array}$ & $\begin{array}{c}15401002 \\
34418 \mathrm{kB}(R=163)\end{array}$ & $\begin{array}{c}14813453 \\
22109 \mathrm{kB}\left(m_{0}=257, m_{1}=11\right)\end{array}$ & $\begin{array}{r}\times 0.998 \\
\times 0.642 \\
\end{array}$ \\
\hline $\begin{array}{c}12477816 \\
983036 \mathrm{kB}(w=19)\end{array}$ & $\begin{array}{c}\mathbf{1 2 1 9 3 2 3 2} \\
119061 \mathrm{kB}(R=1223)\end{array}$ & $\begin{array}{c}12499600 \\
102820 \mathbf{~ k B}\left(m_{0}=1381, m_{1}=7\right)\end{array}$ & $\begin{array}{l}\times 1.025 \\
\times 0.863 \\
\end{array}$ \\
\hline
\end{tabular}

we have used it in a modular exponentiation algorithm which provides memory storage savings or improve the performance in terms of clock cycles per modular exponentiation, while offering a total flexibility in terms of storage amount. We have provided a complexity evaluation, which shows that our approach improves significantly the complexity/storage trade-off. We have then implemented this approach in order to check the performance benefits. We have compared our approach with two other classical approaches, the Fixed-base Comb and the Radix- $R$, and have confirmed the complexity results, showing the better storage/performance trade-off of our approach.

Two issues remain opened:

- Side-channel analysis is also a major threat, even in case of software implementation. For example, Gueron in [7] mentions the cache attack. In the present paper, we did not take this threat into account in the memorization process. However, by using a storage pattern spreading the data in memory, we could ensure the resistance against cache-attacks in the same way as the 
one used by Gueron without penalty. This needs to be implemented in all algorithms for fair comparison.

- Our approach can be applied to Elliptic Curve Cryptography, particularly to the ECDSA signature protocol. In this case, one needs to compute Elliptic Curve Scalar Multiplication. However, the relatively cheap doubling of point operation in comparison with point addition for the NIST recommended curves makes the benefits of our approach not as good as the one in the modular exponentiation case. Therefore, this approach needs to be implemented in relevant curves. For example, the twisted Edwards curve is an example of curve with relatively equivalent doubling and addition in terms of complexity.

\section{References}

1. The GNU Multiple Precision Arithmetic Library (GMP). http://gmplib.org/.

2. Antoon Bosselaers, René Govaerts, and Joos Vandewalle. Comparison of three modular reduction functions. In Advances in Cryptology - CRYPTO '93, Proceedings, pages 175-186, 1993.

3. Acting Secretary Cameron Kerry and USST/Director Patrick Gallagher. Digital Signature Standard (DSS). In Federal Information Processing Standards Publications, volume FIPS PUB 186-4. NIST, 2013.

4. Taher El Gamal. A public key cryptosystem and a signature scheme based on discrete logarithms. In Advances in Cryptology, Proceedings of CRYPTO '84, pages 10-18, 1984.

5. Harvey L. Garner. The residue number system. In Proceedings of the Western Joint Computer Conference, pages 146-153, 1959.

6. Daniel M. Gordon. A survey of fast exponentiation methods. J. Algorithms, 27(1):129-146, 1998.

7. Shay Gueron. Efficient software implementations of modular exponentiation. $J$. Cryptographic Engineering, 2(1):31-43, 2012.

8. Darrel Hankerson, Julio López Hernandez, and Alfred Menezes. Software Implementation of Elliptic Curve Cryptography over Binary Fields. In Cryptographic Hardware and Embedded Systems - CHES 2000, volume 1965 of Lecture Notes in Computer Science, pages 1-24. Springer, 2000.

9. Donald E. Knuth. The Art of Computer Programming, Volume II: Seminumerical Algorithms, 3rd Edition. Addison-Wesley, 1998.

10. Chae Hoon Lim and Pil Joong Lee. More flexible exponentiation with precomputation. In Advances in Cryptology - Crypto '94, volume 839 of Lecture Notes in Computer Science, pages 95-107. Springer, 1994.

11. Lourdes López-García, Luis J. Dominguez Perez, and Francisco RodríguezHenríquez. A pairing-based blind signature e-voting scheme. Comput. J., 57(10):1460-1471, 2014.

12. Peter Montgomery. Modular Multiplication Without Trial Division. Mathematics of Computation, 44(170):519-521, 1985.

13. U.S.D.C. Rebecca Blank and USST/Director Patrick Gallagher. Recommendation for Key Management. In Computer Security, volume Part 1, Rev 3. of NIST Special Publication 800-57, pages 62-64. NIST, 2012.

14. Antonin Svoboda. The numerical system of residual classes in mathematical machines. In IFIP Congress, pages 419-421, 1959. 